\title{
Channel Design and Optimization of Active Transport Molecular Communication
}

\author{
Nariman Farsad ${ }^{1}$, Andrew W. Eckford ${ }^{1}$, and Satoshi Hiyama ${ }^{2}$ \\ 1 Dept. of Computer Science and Engineering, York University \\ 4700 Keele Street, Toronto, ON, Canada M3J 1P3 \\ nariman@cse. yorku.ca, aeckford@yorku.ca \\ 2 Research Laboratories, NTT DOCOMO Inc., Yokosuka, Kanagawa, Japan \\ hiyama@nttdocomo.co.jp
}

\begin{abstract}
In this paper, a guideline is provided for design and optimization of the shape of active transport molecular communication channels. In particular rectangular channels are considered and it is shown that for channels employing a single microtubule as the carrier of the information particles, the smaller the perimeter of the channel, the higher the channel capacity. Furthermore, it is shown that when channels with similar perimeters are considered, square-like channels achieve higher channel capacity for small values of time per channel use, while narrower channels achieve higher information rates for larger values of time per channel use.
\end{abstract}

Key words: Molecular communication, microchannels, information theory.

\section{Introduction}

Molecular communication [1] is a new and emerging form of communication where molecules are used to carry information from a nano- or micro-scale transmitter to a nano- or micro-scale receiver. These information carrying molecules propagate in a fluidic environment we call fluidic microchannels, either passively or actively. In passive transportation, the information molecules diffuse in the channel via Brownian motion from the transmitter to the receiver. In active transport, the information molecules are transported as cargoes using a molecular motor system consisting of kinesins and microtubules.

As shown in $[2,3,4]$, one advantage of active transport over passive transport is the higher degree of freedom in term of design parameters. For example, as shown in [2], parameters such as number of microtubules and the shape of the transmission zone can have a significant effect on the information rate of active transport propagation, where as the effect of such parameters on Brownian motion is nonexistent or minimal. Moreover, in [2] it was shown that active transport propagation can achieve higher information rates than Brownian motion. Therefore in this work we consider active transport propagation of information molecules, and propose elegant techniques for channel design optimization. 
Notable works in this field include a general formulation of molecular communication as a timing channel under Brownian motion $[5,6]$, an analysis of information transfer rates using molecular motors $[7,8]$, mathematical channel models for continuous diffusion [9], binary concentration-encoded molecular communication [10], a simple model comparing the achievable information rates of passive transport using Brownian motion to that of active transport using microtubule filaments moving over a molecular motor track [2, 11], a simple mathematical transport model for active transport propagation [3], and optimization of the transmission zone and vesicular encapsulation process [4]. In $[12,13]$ basic channel design is considered for designing molecular sorters and rectifiers.

Our main contribution in this paper is design optimization of the rectangular channels employing active transport propagation. Typically laborious and time consuming methods relying on engineering intuitions and experimental trial-anderrors are used to design such channels. However in this work we use theoretical techniques to show that when the area of the channel is kept constant, the channel with smallest perimeter (i.e. square channel) maximizes the information transmission rate. When the perimeter is kept constant we show the surprising effect that the square channel achieves higher information rates at small values of time per channel use, while narrower channels achieve higher information rates for larger values of time per channel use.

\section{Active Transport Molecular Communication Environment}

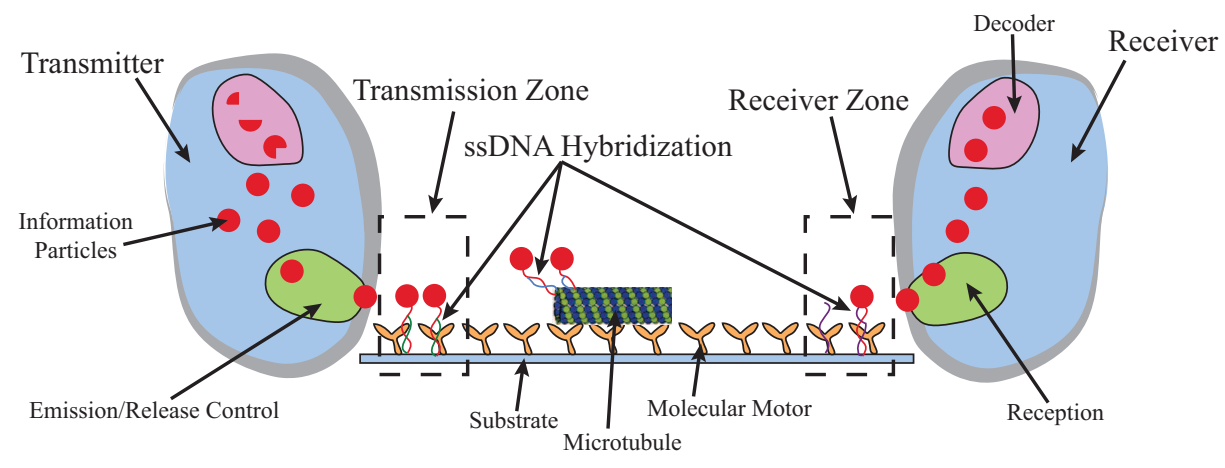

Fig. 1. Depiction of the communication environment.

Our molecular communication environment is similar to the ones in $[2,11,14]$. We use a rectangular propagation environment (with slightly rounded corners), consisting of a transmission zone and a receiver zone. Message-bearing particles 
originate at the transmission zone, and propagate until they arrive at the receiver zone. These particles are initially assumed to be anchored to the transmission zone until microtubule filaments, moving over molecular motor tracks that cover the whole environment, pick up and transport the information carrying particles from the transmission zone to the receiver zone.

As in [14], we assume that the microchannel environment is lined with static kinesin molecular motors, and that these motors cause microtubule filaments to propagate along their surface. The motion of the microtubule is largely regular (compared to pure Brownian motion), although the effects of Brownian motion cause random fluctuations. The loading and unloading mechanics are assumed to be the same as those proposed in [14]. The particles are anchored to the transmission zone through deoxyribonucleic acid (DNA) hybridization bonds, and do not move until they are picked up by a microtubule filament. The pickup and drop-off mechanisms are also assumed to be carried out through DNA hybridization bonds. This process is summarized in Figure 1 and the reader is referred to [14] for detailed explanation.

\subsection{Simulating Active Transport}

Although it has been demonstrated that it is possible to generate and study this molecular communication system in wet labs $[14,15]$, it is very laborious and difficult to study these systems from a communication perspective using laboratory experiments. Therefore, previous works have relied on computer simulations to study these systems. In $[12,13]$ the motion of microtubule filaments over stationary kinesin molecular motors was simulated and in $[2,11]$ a complete simulation of the communication system was presented. In this work, for our simulations we use the same techniques proposed in [2].

Since the microtubules move only in the $x-y$ directions, and do not move in the $z$ direction (along the height of the channel), we consider a two-dimensional simulation of microtubules for $\Delta t$ time intervals. Given some initial position $\left(x_{0}, y_{0}\right)$ at time $t=0$, for any integer $k>0$, the motion of the microtubule is given by the sequence of coordinates $\left(x_{i}, y_{i}\right)$ for $i=1,2, \ldots, k$. Each coordinate $\left(x_{i}, y_{i}\right)$ represents the position of the microtubule's head at the end of the time $t=i \Delta t$, where

$$
\begin{aligned}
x_{i} & =x_{i-1}+\Delta r \cos \theta_{i}, \\
y_{i} & =y_{i-1}+\Delta r \sin \theta_{i} .
\end{aligned}
$$

In this case, the step size $\Delta r$ at each step is an independent and identically distributed (iid) Gaussian random variable with mean and variance

$$
\begin{aligned}
E[\Delta r] & =v_{\text {avg }} \Delta t, \\
\operatorname{Var}[\Delta r] & =2 D \Delta t,
\end{aligned}
$$

where $v_{\text {avg }}$ is the average velocity of the microtubule, and $D$ is the microtubule's diffusion coefficient. The angle $\theta_{i}$ is no longer independent from step to step: instead, for some step-to-step angular change $\Delta \theta$, we have that 


$$
\theta_{i}=\Delta \theta+\theta_{i-1}
$$

Now, for each step, $\Delta \theta$ is an iid Gaussian-distributed random variable with mean and variance

$$
\begin{aligned}
E[\Delta \theta] & =0, \\
\operatorname{Var}[\Delta \theta] & =\frac{v_{\text {avg }} \Delta t}{L_{p}},
\end{aligned}
$$

where $L_{p}$ is the persistence length of the microtubule's trajectory. In [12], these values were given as $v_{\text {avg }}=0.85 \mu \mathrm{m} / \mathrm{s}, D=2.0 \cdot 10^{-3} \mu \mathrm{m}^{2} / \mathrm{s}$, and $L_{p}=111$ $\mu \mathrm{m}$. Following [12], in case of a collision with a boundary, we assume that the microtubule does not reflect off the boundary, as in an elastic collision, but instead sets $\theta_{i}$ so as to follow the boundary.

In order to capture the loading process, in the simulations and the mathematical models, we use the grid loading structure proposed in [2]. For loading an information particle, the microtubule filament must drive close to the anchored particle. Therefore, we divide the transmission zone into a square grid, where the length of each square in the grid is the same as the diameter of the particles. We then distribute particles randomly and uniformly between the squares in the grid. If a microtubule enters a square which is occupied by a particle, we assume the microtubule loads that particle given it has an empty loading slot available. In general, based on laboratory experiments we assume that the microtubules can load multiple particles. For unloading, we assume all the loaded particles are unloaded as soon as a microtubule enters the receiver zone. Figure 2 shows a sample trajectory with the loading and unloading mechanism. Notice that the transmission and the receiver zones in this figure are rectangular strips across the width of the channel.

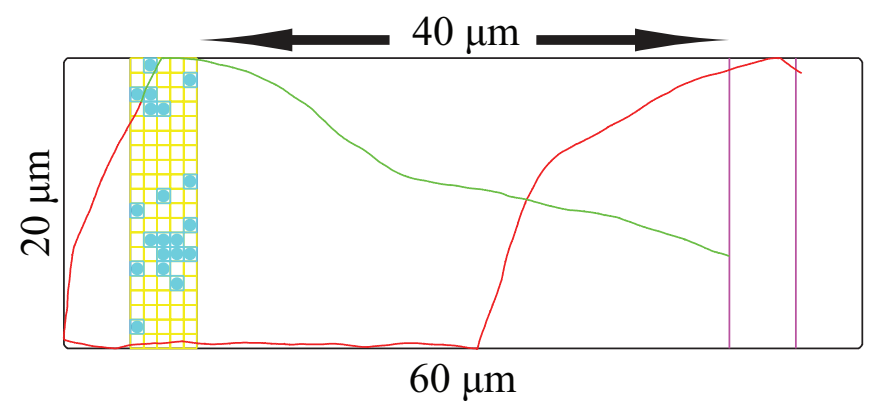

Fig. 2. A sample trajectory of active transport. The path of the microtubule is the line in the middle of the rectangular channel. The red portion corresponds to the path of the unloaded microtubule, while the green portion corresponds to the path of the loaded microtubule. The grid loading structure is on the left hand side and the unloading strip is on the right hand side. The cyan squares in the loading zone represent areas that contain an anchored particle which yellow squares represent areas with no particles. 
In the rest of this paper we assume the channel is rectangular with the transmission zone of the left and the receiver zone on the right side of the channel, as shown in Figure 3. We assume the width and length of the channel, as well as the size of the transmission and the receiver zones, are variable. However, for simplicity we assume the area of the transmission and the receiver zones are the same. Therefore, our rectangular channel environment can be characterized using three parameters: the width of the channel, the length of the channel, and the separation distance between the transmission zone and the receiver zone.

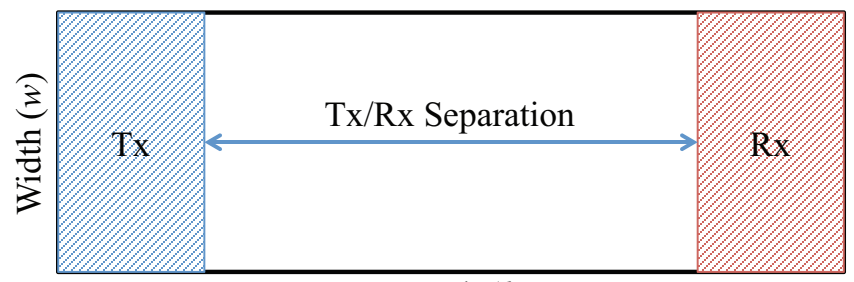

Length $(l)$

Fig. 3. The rectangular molecular communication environment with the transmission zone (blue striped area) on the left and the receiver zone (red striped area) on the right. The size of the transmission and receiver zones are assumed to be the same. The channel is characterized by its width, length and the separation between the transmission and the receiver zones.

\section{Information Rate and Transport Model}

In this section we formulate the channel capacity of molecular communication channel as well as a transport model, all of which will be used to optimize the design of the channel in the next section. Previous work has considered molecular communication either as a timing channel problem (i.e., where information is encoded in the times when molecules are released) [5, 8]; as an inscribed matter problem (i.e., where information is encoded by transmitting custom-made particles, such as specific strands of DNA) [16]; or as a mass transfer problem (i.e., a message is transmitted by moving a number of particles from the transmitter to the receiver) $[2,8,10]$. In this paper, we consider information transmission as a mass transfer problem.

In the simplest possible conception of this scheme, the particles themselves are not information-bearing, and a message is conveyed in the number of particles released by the transmitter. For example, if a maximum of three particles may be used, from a traditional communication system perspective, we may form messages two bits long (i.e., $\left.\log _{2} 4\right)$ : "00" for 0 particles, "01" for 1 particle, "10" for 2 particles, and "11" for 3 particles. However, this message might not be perfectly conveyed to the receiver: given a time limit $T$ for the communication 
session, it is possible that some of the particles will not arrive at the receiver after $T$ has elapsed.

Since we have assumed that messages are encoded in the number of particles, let $X \in \mathcal{X}$ represent the number of particles released into the medium by the transmitter (i.e. the symbol transmitted by the source), $Y \in \mathcal{Y}$ represent the number that arrive at the destination (i.e. the symbol received at the destination) once $T$ seconds have elapsed, and $x_{\max }$ be the maximum number of particles the transmitter can release per channel use. In other words, the set of possible transmission symbols and the set of possible received symbols are $\mathcal{X}=\mathcal{Y}=$ $\left\{0,1,2, \cdots, x_{\max }\right\}$. From the channel's perspective, $X \in \mathcal{X}$ is a discrete random variable given by probability mass function (PMF) $f_{X}(x), Y \in \mathcal{Y}$ is also a discrete random variable given by $\operatorname{PMF} f_{Y}(y)$, and a channel use is defined as $T$ second intervals between the transmission releases.

The maximum rate at which any communication system can reliably transmit information over a noisy channel is bounded by a limit called channel capacity [17]. The channel capacity can be calculated as,

$$
C=\max _{f_{X}(x)} I(X ; Y),
$$

where $I(X ; Y)$ is the mutual information between $X$ and $Y$. Mutual information is defined as

$$
I(X ; Y)=E\left[\log _{2} \frac{f_{Y \mid X}(y \mid x)}{\sum_{x} f_{Y \mid X}(y \mid x) f_{X}(x)}\right],
$$

where, $f_{Y \mid X}(y \mid x)$ represents the probability of receiving symbol $y$ at the destination, given that symbol $x$ was transmitted by the source; $f_{X}(x)$ represents the probability of transmitting symbol $x$; and $E[\cdot]$ represents expectation.

In general, if PMF $f_{Y \mid X}(y \mid x)$ is known, we can calculate mutual information for any $f_{X}(x)$. However, in order to calculate the channel capacity, we need to find the PMF $f_{X}(x)$ that maximizes mutual information. We can use the BlahutArimoto algorithm $[18,19]$ to find the $\operatorname{PMF} f_{X}(x)$ such that, given $f_{Y \mid X}(y \mid x)$, mutual information is maximized. Therefore, if PMF $f_{Y \mid X}(y \mid x)$ is known, we can calculate the channel capacity of the molecular communication system in a straightforward manner.

In $[3,4]$, a simple transport model is presented to estimate the PMF $f_{Y \mid X}(y \mid x)$ in a computationally efficient manner. This transport model also provides the necessary insight into the underlying mechanics effecting $f_{Y \mid X}(y \mid x)$. According to this model, the PMF $f_{Y \mid X}(y \mid x)$ depends on two random variables: a Bernoulli random variable $V_{i}$, representing the event that the $i$ th square is visited in a single microtubule trip from the transmitter to the receiver, and a random variable $K$ representing the number of microtubule trips between the transmitter and the receiver during the time interval $T$. In $[3,4]$ the random variable $V_{i}$ was considered for optimization of the shape of the transmission zone. In this work, we study the effects of channel size on these random variables and use them to optimize the design. 


\section{Design Analysis}

In this section, we consider the design analysis of rectangular microchannels to maximize their channel capacity. In particular, we consider properties of rectangular channels such as area and perimeter as optimization criterion. We separate the effects of area and perimeter by considering two scenarios: 1) we keep the area constant and change the perimeter; and 2) we keep the perimeter constant and change the area of the rectangular channel. Table 1 summarizes the sample channels with constant area, and Table 2 shows the sample channels with constant perimeter.

Table 1. Channels with constant area and different perimeters

\begin{tabular}{cccc}
\hline \hline Dimensions $(w \times l)$ & Tx/Rx Separation & Area $(\mathrm{A})$ & Perimeter $(\mathrm{P})$ \\
\hline $5 \mu \mathrm{m} \times 240 \mu \mathrm{m}$ & $160 \mu \mathrm{m}$ & $1200 \mu \mathrm{m}^{2}$ & $490 \mu \mathrm{m}$ \\
$10 \mu \mathrm{m} \times 120 \mu \mathrm{m}$ & $80 \mu \mathrm{m}$ & $1200 \mu \mathrm{m}^{2}$ & $260 \mu \mathrm{m}$ \\
$20 \mu \mathrm{m} \times 60 \mu \mathrm{m}$ & $40 \mu \mathrm{m}$ & $1200 \mu \mathrm{m}^{2}$ & $160 \mu \mathrm{m}$ \\
$40 \mu \mathrm{m} \times 30 \mu \mathrm{m}$ & $20 \mu \mathrm{m}$ & $1200 \mu \mathrm{m}^{2}$ & $140 \mu \mathrm{m}$ \\
\hline
\end{tabular}

Table 2. Channels with constant perimeter and different areas

\begin{tabular}{cccc}
\hline \hline Dimensions $(w \times l)$ & Tx/Rx Separation & Area $(\mathrm{A})$ & Perimeter $(\mathrm{P})$ \\
\hline $10 \mu \mathrm{m} \times 70 \mu \mathrm{m}$ & $30 \mu \mathrm{m}$ & $700 \mu \mathrm{m}^{2}$ & $160 \mu \mathrm{m}$ \\
$20 \mu \mathrm{m} \times 60 \mu \mathrm{m}$ & $40 \mu \mathrm{m}$ & $1200 \mu \mathrm{m}^{2}$ & $160 \mu \mathrm{m}$ \\
$30 \mu \mathrm{m} \times 50 \mu \mathrm{m}$ & $36 \mu \mathrm{m}$ & $1500 \mu \mathrm{m}^{2}$ & $160 \mu \mathrm{m}$ \\
$40 \mu \mathrm{m} \times 40 \mu \mathrm{m}$ & $30 \mu \mathrm{m}$ & $1600 \mu \mathrm{m}^{2}$ & $160 \mu \mathrm{m}$ \\
\hline
\end{tabular}

In the next two subsections we use these two sets of channels to analyse the effects of channel size on the number of microtubule trips and the probability that squares in the grid loading area are visited.

\subsection{Constant Area}

If the area of the rectangular channel is kept constant, increasing the perimeter yields longer and narrower channels. Since the microtubules mostly follow the walls of the channel, the larger the perimeter the smaller the average value of $K$ (i.e. the average number of microtubule trips during time interval $T$ ). This effect can be seen by comparing the probability density function (PDF) of the $5 \mu \mathrm{m} \times 240 \mu \mathrm{m}$ and the $40 \mu \mathrm{m} \times 30 \mu \mathrm{m}$ channels as shown in Figure 4 for time per channel use of 750 seconds. There are zero microtubule trips for the longer and narrower channel $(5 \mu \mathrm{m} \times 240 \mu \mathrm{m})$, while there are between 1 and 4 microtubule trips for the square-like channel $(40 \mu \mathrm{m} \times 30 \mu \mathrm{m})$. 

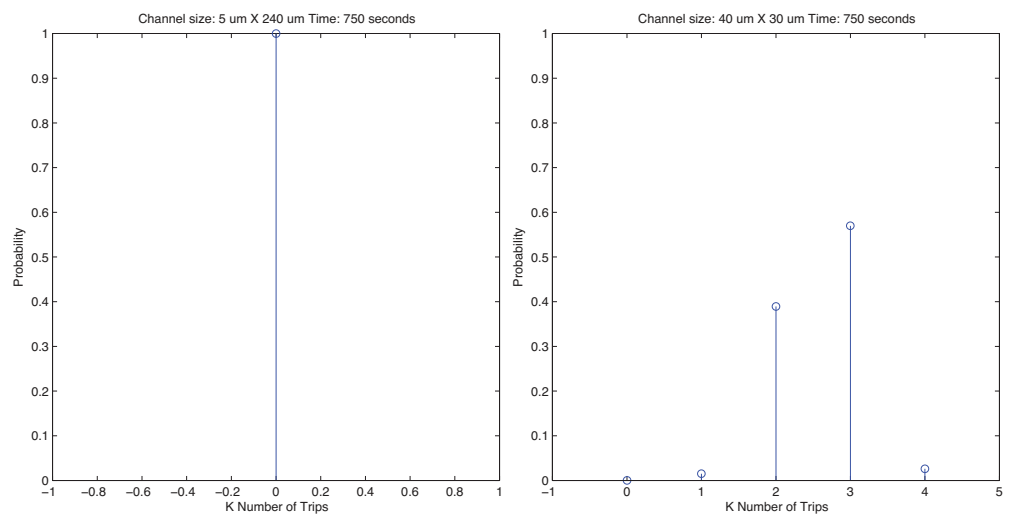

Fig. 4. Probability distribution function of $K$.

In contrast, as the channel gets narrower the probability that a square in the grid is visited is increased, because a larger number of squares are close to the walls. In $[3,4]$ it was shown that the squares that are closer to the walls of the channel have a much higher probability of being visited in a single microtubule trip. However, the number of trips a microtubule takes between the transmitter and the receiver over time duration $T$ is a more dominant factor. This is because the molecular communication is a mass transfer problem, which depends on the microtubules. Therefore, we would expect that keeping the area constant the channel is optimized when the perimeter is smallest (i.e. the channel is a square). This effect will be shown in the results section.

\subsection{Constant Perimeter}

If the perimeter of the rectangular channel is kept constant, the average number of trips a microtubule travels during time interval $T$ seconds are almost identical regardless of the dimensions of the channel. This effect is due to the fact that microtubules follow the walls of the channel with a high probability, as shown in [4]. However, as the channel becomes narrower the number of trips becomes more deterministic, while as the channel gets closer to a square the number of trips become more random (i.e. the variance of $K$ decreases as the channel gets narrower, while the variance increases when the channel is closer to a square). This effect can be seen in Figure 5 where the PDF of $K$ is plotted over time duration of 750 seconds for the $10 \mu \mathrm{m} \times 70 \mu \mathrm{m}$ and the $40 \mu \mathrm{m} \times 40 \mu \mathrm{m}$ channels. While that average number of trips in both channels is close to 2 , for the variance of $K$ is lower for the narrower channel compared to the square channel.

Another interesting and surprising effect is that for small values of time duration per channel use, the higher variance of the square channels are constructive. For example, from Figure 6 we can see that for time durations of 250 seconds, there is a higher chance of a single microtubule trip between the transmitter and 

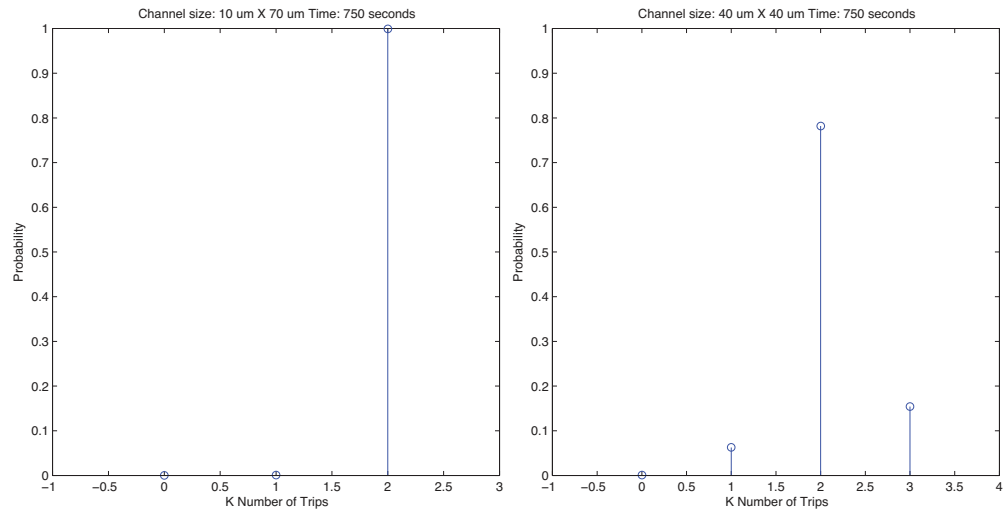

Fig. 5. Probability distribution function of $K$.

the receiver for a square-like channel compared to a narrower channel. Therefore, for small time durations we would expect the square-like channels to be better and for larger time durations the narrower channel.
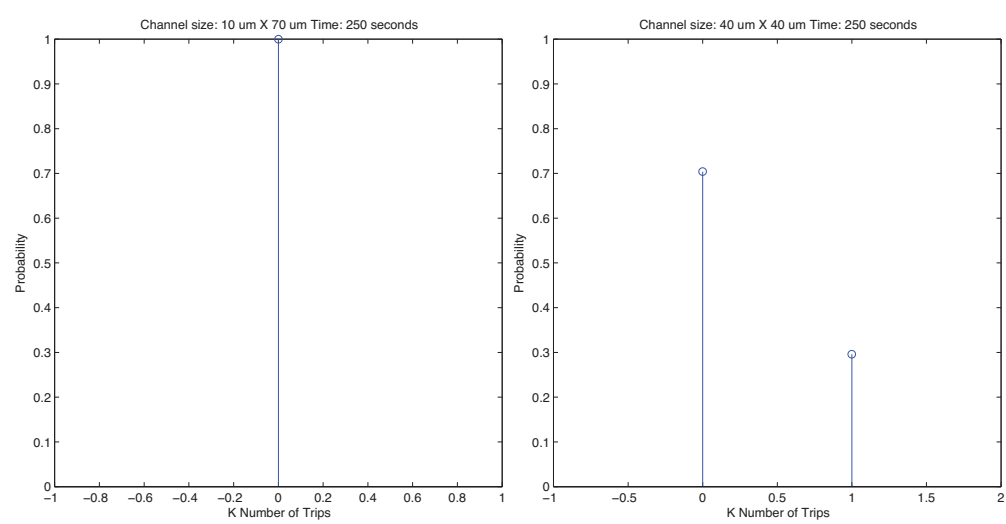

Fig. 6. Probability distribution function of $K$.

\section{Results}

Figure 7 shows the channel capacity in bits versus $x_{\max }$ for different values of time per channel use. From the results we can see that channels with smaller perimeter generally achieve higher information rates because the microtubules can travel a larger number of times between the transmitter and the receiver. 
Moreover, we can see that for small time per channel use values when the channels have similar perimeters, the square-like channels perform better because there is a higher chance that the microtubules travels once between the transmitter and the receiver. However when perimeter is kept constant, as time per channel use increases, the narrower channels achieve higher information rates.
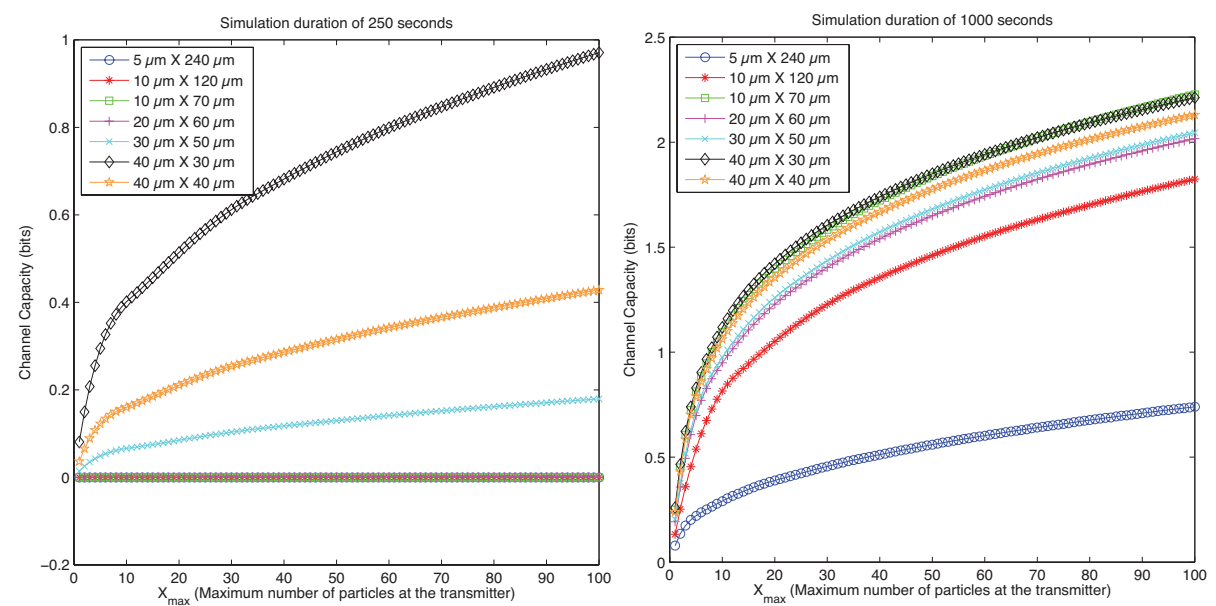

Fig. 7. Channel capacity versus $x_{\max }$.

\section{Conclusion and Future Works}

In this work we presented a theoretical design and optimization of rectangular molecular communication channels employing active transport propagation. In particular, we showed that for channels employing a single microtubule as the carrier of the information particles, the smaller the perimeter of the channel, the higher the channel capacity. Furthermore, we showed that when channels with similar perimeters are considered, square-like channels achieve higher channel capacity for small values of time per channel use, while narrower channels achieve higher information rates for larger values of time per channel use.

As part of the future work we will consider channels with multiple microtubules. In these channels as the area of the channel increases, the number of microtubules assisting in transmission increases. Using the results from this paper, and the fact that lager channel areas can accommodate larger number of microtubules, we want to maximize area while we minimize the perimeter. Solving this optimization problem will result in an optimal channel. Because circle has the largest area to perimeter ratio, we will also consider non-rectangular channels such as circular and ring-shaped channels. 


\section{References}

1. S. Hiyama and Y. Moritani, "Molecular communication: harnessing biochemical materials to engineer biomimetic communication systems," Nano Communication Networks, vol. 1, no. 1, pp. 20-30, 2010.

2. N. Farsad, A. W. Eckford, S. Hiyama, and Y. Moritani, "Information rates of active propagation in microchannel molecular communication," in Proc. of the 5th International ICST Conference on Bio-Inspired Models of Network, Information, and Computing Systems, (Boston, MA), p. 7, 2010.

3. N. Farsad, A. W. Eckford, S. Hiyama, and Y. Moritani, "A simple mathematical model for information rate of active transport molecular communication," in Proc. IEEE INFOCOM Workshops, (Shanghai, P. R. China), pp. 473-478, 2011.

4. N. Farsad, A. W. Eckford, S. Hiyama, and Y. Moritani, "Quick system design of vesicle-based active transport molecular communication by using a simple transport model," Nano Communication Networks, 2011. DOI: 10.1016/j.nancom.2011.07.003.

5. A. W. Eckford, "Nanoscale communication with brownian motion," in Proc. of the 41st Annual Conference on Information Sciences and Systems, (Baltimore, MD), pp. 160-165, 2007.

6. B. Atakan and O. Akan, "An information theoretical approach for molecular communication," in Proc. of the 2nd International Conference on Bio-Inspired Models of Network, Information and Computing Systems, (Budapest, Hungary), pp. 33-40, 2007.

7. M. J. Moore, T. Suda, and K. Oiwa, "Molecular communication: modeling noise effects on information rate," IEEE Transactions on NanoBioscience, vol. 8, no. 2, pp. 169-180, 2009.

8. A. W. Eckford, "Timing information rates for active transport molecular communication," in Nano-Net, vol. 20 of Lecture Notes of the Institute for Computer Sciences, Social Informatics and Telecommunications Engineering, pp. 24-28, Springer Berlin Heidelberg, 2009.

9. M. Pierobon and I. F. Akyildiz, "A physical end-to-end model for molecular communication in nanonetworks," IEEE Journal on Selected Areas in Communications, vol. 28, no. 4, pp. 602-611, 2010.

10. M. U. Mahfuz, D. Makrakis, and H. T. Mouftah, "On the characterization of binary concentration-encoded molecular communication in nanonetworks," Nano Communication Networks, vol. 1, no. 4, pp. 289-300, 2010.

11. A. W. Eckford, N. Farsad, S. Hiyama, and Y. Moritani, "Microchannel molecular communication with nanoscale carriers: Brownian motion versus active transport," in Proc. of the IEEE International Conference on Nanotechnology, (Seoul, South Korea), pp. $854-858,2010$.

12. T. Nitta, A. Tanahashi, M. Hirano, and H. Hess, "Simulating molecular shuttle movements: Towards computer-aided design of nanoscale transport systems," Lab on a Chip, vol. 6, no. 7, pp. 881-885, 2006.

13. T. Nitta, A. Tanahashi, and M. Hirano, "In silico design and testing of guiding tracks for molecular shuttles powered by kinesin motors," Lab on a Chip, vol. 10, no. 11, pp. 1447-1453, 2010.

14. S. Hiyama, R. Gojo, T. Shima, S. Takeuchi, and K. Sutoh, "Biomolecular-motorbased nano- or microscale particle translocations on dna microarrays," Nano Letters, vol. 9, no. 6, pp. 2407-2413, 2009. 
15. S. Hiyama, Y. Moritani, R. Gojo, S. Takeuchi, and K. Sutoh, "Biomolecular-motorbased autonomous delivery of lipid vesicles as nano- or microscale reactors on a chip," Lab on a Chip, vol. 10, no. 20, pp. 2741-2748, 2010.

16. L. C. Cobo and I. F. Akyildiz, "Bacteria-based communication in nanonetworks," Nano Communication Networks, vol. 1, no. 4, pp. 244-256, 2010.

17. T. M. Cover and J. A. Thomas, Elements of Information Theory 2nd Edition. Wiley-Interscience, 2 ed., 2006.

18. R. Blahut, "Computation of channel capacity and rate-distortion functions," IEEE Transactions on Information Theory, vol. 18, no. 4, pp. 460-473, 1972.

19. S. Arimoto, "An algorithm for computing the capacity of arbitrary discrete memoryless channels," IEEE Transactions on Information Theory, vol. 18, no. 1, pp. 1420, 1972 . 